\title{
Triazolinedione bearing gels
}

\author{
Saltuk B. Hanay
}

Faculty of Engineering and Natural Sciences, Sabanci University, 34956, Tuzla, Istanbul, Turkey.

ABSTRACT: Triazolinediones (TADs) have been extensively used for the modification and crosslinking of polymers. However, there have not been any report on TAD bearing gels so far except an inorganic silica gel on which TAD is immobilized via ionic bonding. Here, I report a simple, scalable and general strategy to synthesize gels that contains covalently bonded TAD groups, starting from commercially available poly(hexamethylene diisocyanate). The covalently bonded TAD-gel platform could find many different applications such as the fabrication of hybrid materials, chemo-sensing, and scavenging excess reagents. In this work, potential scavenging application for TAD-gels were demonstrated with three different compound furan, aniline and limonene. Moreover, 1naphthol was selectively and completely removed from its mixture with 2-naphthol. The strategy developed here enables the preparation of poly(urea) and poly(ureathane) based novel materials via ultrafast modification at TAD moieties.

Triazolinediones (TADs) are exceptionally reactive compounds with a varying degree of stability - from minutes to months - depending on their substructure. Recently, they have become very popular among polymer chemists due to their ultrafast reactions which does not require UV, heat or a chemical initiator. Simple mixing of the reagents at ambient temperature is generally sufficient for TAD reactions.

To date, TADs have been used in many different areas such as Diels-Alder reactions, ${ }^{1}$ homopolymerization, ${ }^{2}$ step growth polymerization, ${ }^{3}$ modification of polydienes, ${ }^{4}$ protection and deprotection of indole's $\pi$-bond, ${ }^{5}$ fluorescent labeling of vitamin $\mathrm{D},{ }^{6}$ scavenging dienes, ${ }^{7,8}$ ultrafast preparation of multilayer films, ${ }^{9}$ modification of amino acids - furyl-alanine, ${ }^{10}$ tyrosine, ${ }^{11-17}$ tryptophan ${ }^{18}$ — and fabrication of poly(amino acid) based hybrid gels ${ }^{19}$. Moreover, some TAD reactions are reversible and this property was exploited to create self-healing dynamic materials, ${ }^{20}$ light-responsive compounds ${ }^{21}$ and light stabilized materials. ${ }^{22}$ Many other uses of TADs and TAD-related materials have been reported in the literature as well. ${ }^{23}$ However, there has not been any reports of TAD-bearing gels. TADgels could be extremely advantageous since a variety of functional groups can be attached to these gels in an ultrafast and 'click-like' manner. Therefore, these novel gels can find use in many different applications such as purification and separation of mixtures, sensing analytes, fabrication of polyurethanepoly(amino acid) platforms and for the design of hybrid materials with self-healing capability. In this work, I report a simple preparation method to obtain TAD-bearing gels from commonly available reagents. Moreover, I demonstrate their potential as possible scavengers for Diels-Alder, electrophilic aromatic substitution (EAS) and Alder-ene reactions.

Previously, Keana and co-workers reported silica gel bound TAD by the reaction between custom synthesized sulfonated 4Aryl-TADs and amine bearing silica gels. ${ }^{7}$ In this system, a 0.4 mmol active TAD was present for $1 \mathrm{~g}$ of silica-TAD as quantified by titration. They showed that silica-TAD material can be used to separate diene containing ergosterol from cholesterol mixture. The scavenging potential of TAD was also demonstrated in a solution, rather than as solid-supported TAD, by Werner and Curran where they synthesized several fluorous dienophiles and thereby removed excess dienes in Diels-Alder reaction. ${ }^{8}$ Reacted fluorous dineophiles were removed by filtering over fluorous silica gel. Among all the tested dienophiles, TAD derivatives showed superior reactivity. Inspired by these

Scheme 1. Synthesis of triazolinedione bearing gel
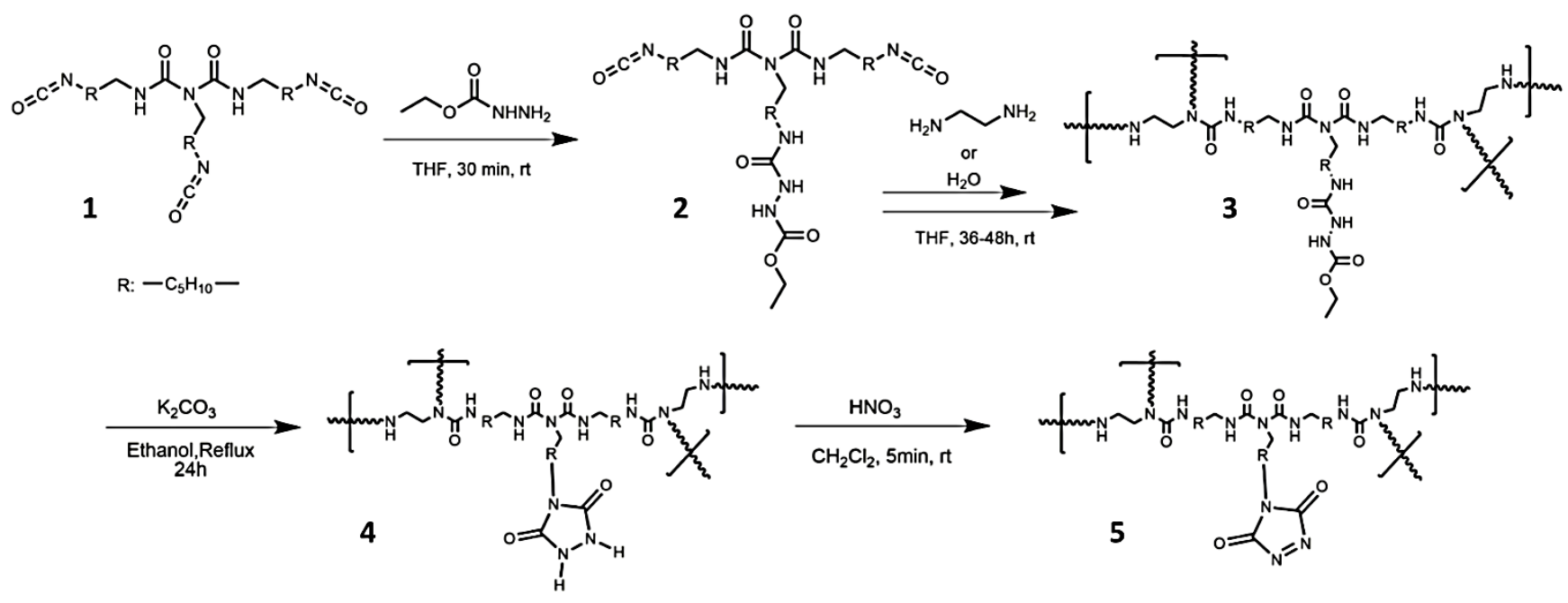
studies, I demonstrated the practicality of TAD-gels reported here to simplify the scavenging process. In terms of practicality, TAG-gels are easy to synthesize unlike silica-TAD, and does not require special fluorous column for filtration unlike fluorous TAD scavengers. Additionally, TAD content of the gels reported here was relatively higher compared to silica-TAD - up to $0,62 \mathrm{mmol}$ of TAD per gram gel.

A trifunctional isocyanate (1) poly(hexamethylene diisocyanate) was chosen as the starting material (Scheme 1). Then, either 1 or $1.5 \mathrm{~mol}$ of isocyanate groups were reacted with an equimolar of ethyl carbazate to form isocyanate-semicarbazide containing intermediate (2). In the same reaction flask, this intermediate was indirectly polymerized via addition of water. Eventually, polymerization of poly(urea) was accompanied with gelation (3). Semicarbazide containing poly(urea) gel (3) was cyclized into urazole containing gel (4) under basic conditions. Finally, the oxidation of urazole containing gel by $\mathrm{HNO}_{3}$ resulted in TAD bearing gel (5). The synthetic procedure and a video summarizing all steps are available as Supporting Information.

\section{Results and Discussion}

TAD formation can be monitored by naked eye owing to the characteristic red/pink color of TAD compounds (Figure $1 \mathrm{a}-\mathrm{b}$ ). Moreover, FTIR analysis revealed the occurrence of a new carbonyl mode at $1765 \mathrm{~cm}^{-1}$ and increase in $\mathrm{C}-\mathrm{N}$ mode at 1360 $\mathrm{cm}^{-1}$ induced in the structure owing to $\mathrm{N}=\mathrm{N}$ formation. An unknown mode appeared at $729 \mathrm{~cm}^{-1}$ but it might be related to gel backbone- $\mathrm{HNO}_{3}$ interaction as it did not change after TAD reaction or decomposition. Scavenging experiments, as discussed below, further verifies the TAD origin of these gels.

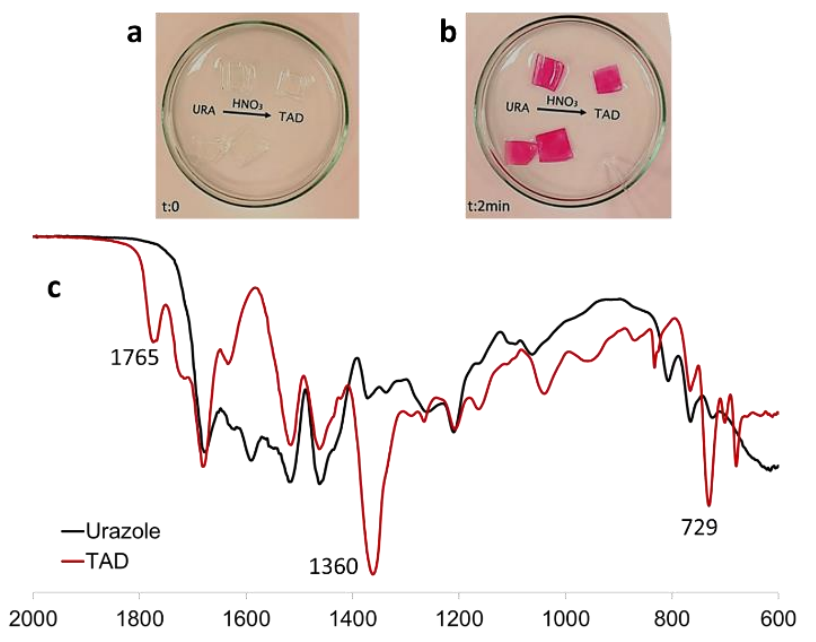

Figure 1. a) Urazole bearing gels placed into dichloromethane containing glass petri dish b) 2 minutes after $100 \mu \mathrm{l}$ of $\mathrm{HNO}_{3}$ was added to urazole gel c) FT-IR spectrum of urazole bearing gel (neat) and its oxidized form TAD bearing gel (neat).

Stabilities of the synthesized TAD gels were tested in different solvents (Figure 2). Approximately $100 \mathrm{mg}$ of TAD-bearing gels were placed into $1.5 \mathrm{~mL}$ solvents in test tubes. Then, the tubes were shaken for 20-30 seconds and the stabilities of gels were monitored through discoloration. For most of the solvents, the TAD-gels were active up to 40 minutes, which is much longer than the duration of typical TAD reactions. Trifluoroacetic acid was found to be incompatible with the material. After 2-3 minutes, popping sounds and turbidity were observed. Gels were quite stable in ethyl acetate, acetonitrile and tetrahydrofuran. In other solvents, TADs barely survived for an hour. DMSO and DMF caused deformation in gels after 24 hours. In other solvents, no deformation was observed. When the TADgel was kept as a solid at ambient conditions, pink color of gels disappeared in 5 hours at room temperature. However, TAD activity remained intact at least a week at $-20{ }^{\circ} \mathrm{C}$.

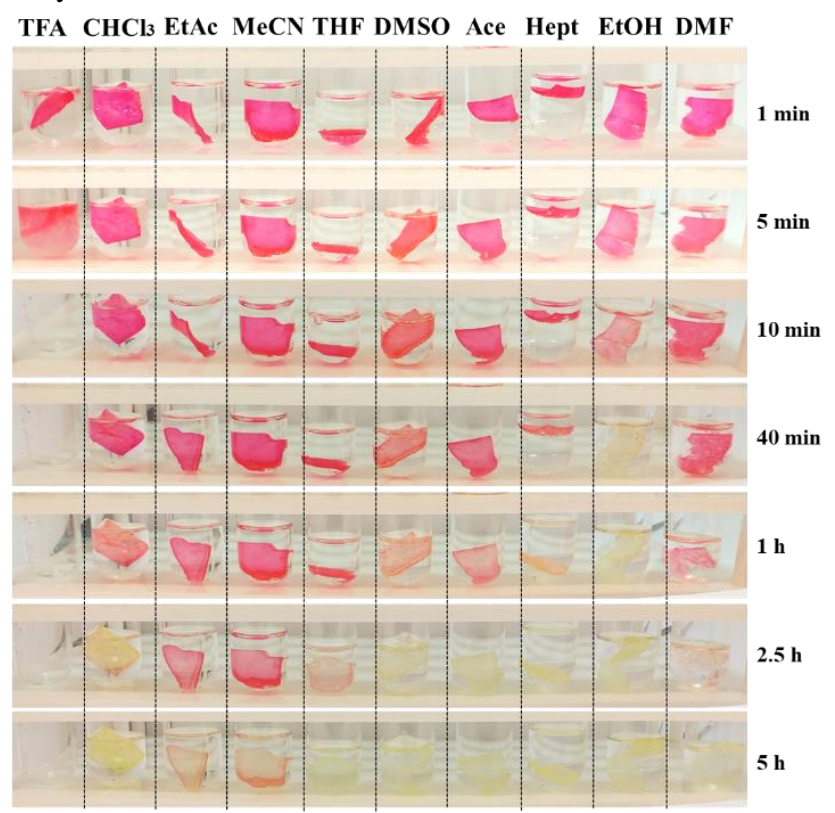

Figure 2. Stability of TAD-gels in different solvents. Detailed information about solvents are listed in experimental section.. Solvents left to right: Trifluoroacetic acid, chloroform, ethyl acetate, acetonitrile, tetrahydrofuran, dimethyl sulfoxide, acetone, heptane, ethanol, N,N-dimethylformamide. TFA was stopped after 5 minutes due to incompatibility

As the decomposition mechanism, water content of the solvents was considered as the culprit since it is well known that water decomposes TAD moieties gradually over time. However, gels in solvent-grade heptane and heptane dried by molecular sieves showed very similar decomposition times. This observation indicates that gel decomposition was either dominated by other factors, or by the absorbed water coming from $\mathrm{HNO}_{3}$. As for other possible decomposition mechanisms, free amines, biuret groups or the TAD self-dimerization may decompose TAD groups over time. Further studies can be conducted to elucidate the underlying mechanism, such as exploring different oxidation methods, and changing synthesis pathway to obtain poly(uretheane), rather than poly(urea).

Scavenging capabilities of TAD gels were tested with different compounds: aniline, limonene and furan. Aniline reacts with TAD via (EAS) whereas limonene via Alder-ene reaction. On the other hand, Furan derivates react commonly via Diels-Alder, but also via EAS ${ }^{10}$. For the experiments, gels were cut into smaller pieces to increase the surface area and to decrease the diffusion time of the reactants into the gel. It was observed that Furan reacted the fastest, whereas aniline and limonene reacted with nearly the same rate, in equimolar concentrations. Approximately in 20 minutes, the red color of TADs completely disappeared in all reactions, whereas no change was observed in the control group (Figure 3). The characterization of the gels was performed by FTIR analysis. For all compounds, $\mathrm{C}=\mathrm{C}-\mathrm{H}$ bending mode appeared at $907 \mathrm{~cm}^{-1}$ and for aniline $\mathrm{C}-\mathrm{NH} 2$ stretching mode was seen at $1300 \mathrm{~cm}^{-1}$ (Figure S2-4). 

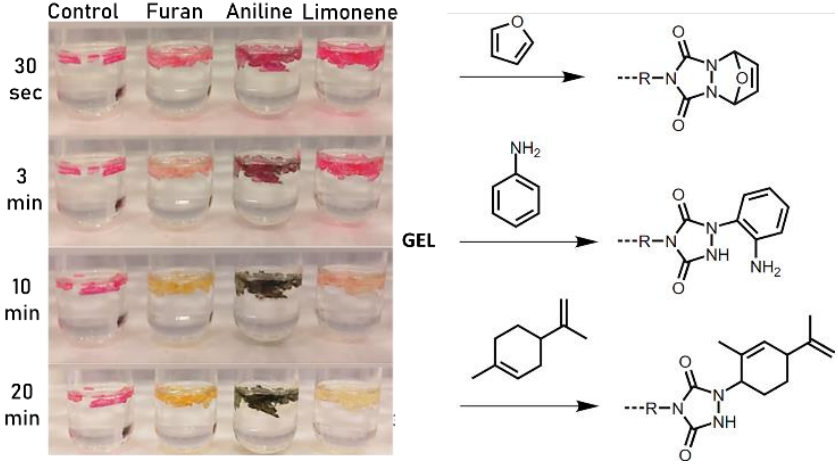

Figure 3. Reaction of TAD-gels with furan, aniline and limonene. The characteristic red color of TAD was disappeared after reaction.

Quantifying active TAD groups on the gels can be done by titration against one of the reactants. However, titration method for this gel is prone to human errors, as reactants take some extra time to diffuse deeper into the gel. On the other hand, NMR analysis may be used to quantify TADs in a more reliable manner. In order to perform NMR quantification, standard solution was prepared in $\mathrm{CDCl}_{3}(6 \mathrm{~mL})$ using furan $(50 \mu \mathrm{l})$ as a reactant and acetonitrile $(50 \mu \mathrm{l})$ as a reference peak for NMR integration. This solution was kept closed at all times in order to prevent the loss of furan as it has low boiling point. $200 \mathrm{mg}$ of TAD gel was added to $\mathrm{CDCl}_{3}$ and NMR spectrum was taken after 10 and 30 minutes. Then, $200 \mathrm{mg}$ more TAD-gel was added. After 60 minutes, red color was disappeared completely. NMR analysis showed a decrease of $36 \%$ for furan peaks. This means, $50 \mu \mathrm{l}$ (x36\%) furan $(18 \mu \mathrm{l}-16.8 \mathrm{mg}-0.283 \mathrm{mmol})$ was reacted with $400 \mathrm{mg}$ of gel. Therefore, $1 \mathrm{~g}$ of TAD gels contains at least 0.62 mmol TAD present. It should also be noted that some active TADs decomposed without reacting with furan which results in a smaller value.

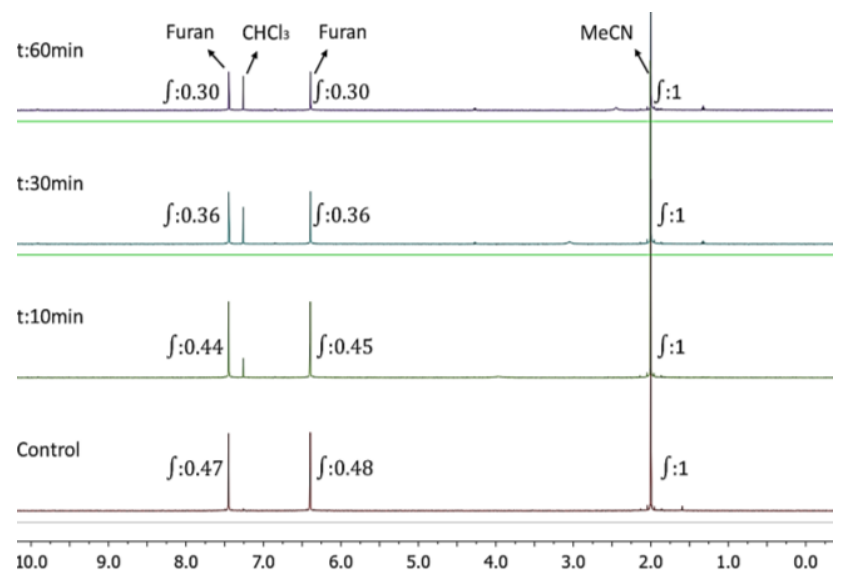

Figure 4. ${ }^{1} \mathrm{H}-\mathrm{NMR}$ spectrum of the TAD-gel scavenged furan and acetonitrile containing $\mathrm{CDCl}_{3}$ stock solution over time. Acetonitrile added as reference for integration to quantify furan loss.

Theoretically, there can be a maximum of $1.65 \mathrm{mmol}$ TAD if equimolar ethyl carbazate and triisocyanate were used at the beginning. It is observed that 1:1.5 (triisocyanate:ethyl carbazate) could also form crosslinked gels. Therefore, there is still space to optimize oxidation conditions and explore different formulations to obtain higher active TAD content.
After demonstrating scavenging potential with individual molecules, a more realistic and challenging scavenging test was performed. Here, an equimolar mixture of 1-naphtol and 2naphtol was treated with TAD-gel. 1-naphtol could react with TAD-gel via electrophilic aromatic substitution or 5-8 adduct formation. On the other hand, 2-naphtol could also react via 14 adduct formation. NMR studies indicated that TAD-gel selectively reacted with 1-naphtol (Figure 5). After 15 minutes, 1naphtol was almost completely removed from the solution, yet almost no change was observed in the 2-naphtol peak. One hour after the treatment, a decrease in 2-naphtol peak was observed which indicates a reaction between TAD-gel and 2-naphtol. The results demonstrate that TAD-gel have potential to be used as a selective scavenger for mixtures.

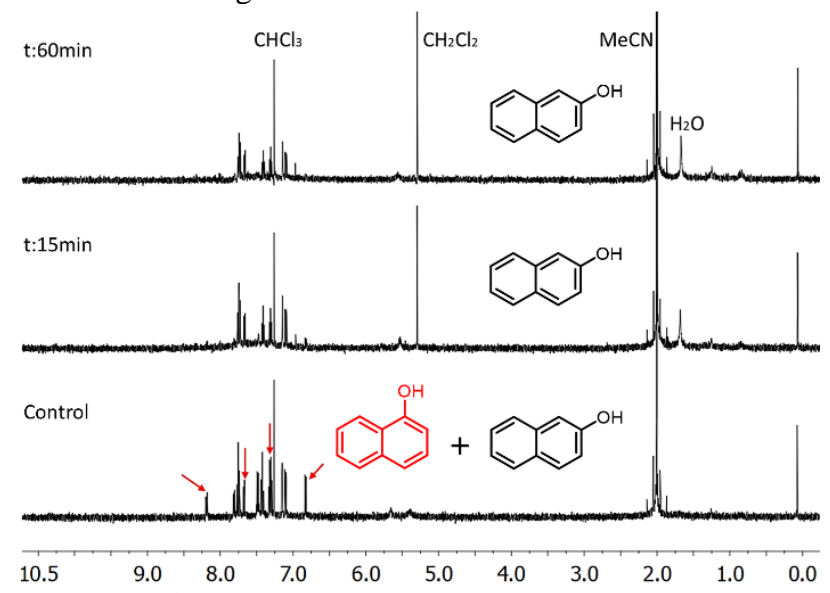

Figure 5. ${ }^{1} \mathrm{H}-\mathrm{NMR}$ spectrum of the TAD-gel scavenged 1naphthol and 2-naphthol mixture. Peaks at 8.18, 7.82, 7.49, $7.47,7.43,6.83 \mathrm{ppm}$ were disappeared after soaking TAD-gel into the solution.

TAD-gels can easily be synthesized in large scale from commonly available and low-cost reagents. As an alternative to the route described here with trifunctional isocyanates, difunctional isocyanates could also be used together with water or multi amine linkers to synthesize similar TAD gels by tuning the stoichiometric ratios of ethyl carbazate/diisocyanate and multiamine containing linkers. Moreover, rigid or elastomeric isocyanates could also be used as comonomers by just adding them after the first step of the synthesis.

The decomposition of TAD-gels might seem as a limitation at first glance; however, the ultrafast reactivity of TADs ensures that the reactions proceed much faster than the decomposition. In general, TAD reactions finish within seconds or several minutes. Therefore, the relatively slow nature of decomposition renders problems associated with decomposition insignificant for practical purposes. On the other hand, the precursor of TAD gel - urazole gel - is very stable. Urazole gel can be stored for months and readily oxidized into TAD-gels whenever desired. As mentioned before, poly(ureathane) based TAD gels can also be synthesized and their stability is expected to be even better since amine formation in poly(urea) is thought to be one factor for the decomposition of TADs. To change the route, polyols - instead of water - could be used in the second step of the synthesis.

The oxidation of urazole to TAD could be carried out in many alternative ways. Here, $\mathrm{HNO}_{3}$ used as an oxidizer as it is readily available and also can be easily washed away. However, care must be taken with $\mathrm{HNO}_{3}$ incompatibilities such as with aniline. 
Therefore, gels have to be washed well to get rid of $\mathrm{HNO}_{3}$. Additionally, water from $\mathrm{HNO}_{3}$ will gradually decompose TAD. Interestingly, some oxidation methods such as electrooxidation or photo-oxidation could be employed to create patterned TAD-gels from urazole gels. Alternatively, an oxidizer solution could also be used to create patterns via inkjet printing.

In conclusion, I reported an easy way to prepare TAD containing gels. These new gels can be used to prepare poly(urea) or poly(ureathane) based novel materials. Among many possible application areas, scavenging excess reagents was tested. It was shown that TAD-gel can be used as scavenger for different reactions and variety of functional groups.

\section{ASSOCIATED CONTENT}

Electronic Supporting Information and gel synthesis video are available.

\section{AUTHOR INFORMATION}

\section{Author Contributions}

All of the work done by Saltuk B. Hanay.

\section{Funding Sources}

Author would like to thank Sabanci University's FENS Fellowship Program for funding.

\section{ACKNOWLEDGMENT}

\section{REFERENCES}

1. Cookson, R. C.; Gilani, S. S. H.; Stevens, I. D. R. Tetrahedron Lett. 1962, 3,615-618. 1461

2. Lai, Y., Butler, G. B., J. Macromol. Sci. Chem, 1985, 10, $1443-$

3. Mallakpour, S. E.; Asghari, J.; Schollmeyer, D. Polym. Int. 1996, $41,43-52$

4. Butler, G. B., Ind. Eng. Chem. Prod. Res. Dev., 1980, 19, 4,512 528

5. Baran, P. S., Guerrero, C. A., Corey, E. J., Org. Lett., 2003, 5, 11 , 1999-2001

6. Shimizu, M., Yamazaki, T., Yamada, S., Bioorg. Med. Chem.,1993, 3, 9, 1809-1814

7. Keana, J. F. W.; Guzikowski, A. P.; Ward, D. D.; Morat, C.; Van Nice, F. L. J. Org. Chem. 1983, 48, 2654-2660.
8. Werner, S.; Curran, D. Org. Lett. 2003, 5, 3293-3296

9. Vonhören, B., Roling, O., De Bruycker, K., Calvo, R., Du Prez,

F. E., Ravoo, B. J., ACS Macro Lett., 2015, 4 (3), 331-334

10.Hoogewijs, K.; Buyst, D.; Winne, J. M.; Martins, J. C.; Madder,

A. Chem. Commun. 2013, 49, 2927-2929

11. Ban, H.; Gavrilyuk, J.; Barbas, C. F., J. Am. Chem. Soc. 2010, $132,1523-1525$.

12. Bauer, D. M., Ahmed, I., Vigovskaya, A., Fruk, L. Bioconjug Chem. 2013, 24, 6, 1094-1101

13. Adamo, R., Allen, M., Berti, F., Danieli, E., Hu, Q.Y., US Patent 9149541B2, 2012

14. Ban, H. Nagano, M., Gavrilyuk, J., Hakamata, W., Inokuma, T., Barbas III, C. F., Bioconjug. Chem. 2013, 24 (4), 520-532.

15. Hanay, S. B., Ritzen, B., Brougham, D., Dias, A. A., Heise, A., Macromol. Biosci., 2017, 17, 7, 1700016.

16. Vandewalle, S., De Coen, R., De Geest, B., Du Prez, F. E., ACS Macro Lett, 2017, 6, 12, 1368-1372

17. Jessica, F., Corentin, W., Sylvestre, D., Christian, L., André, L. RSC Advances, 2013, 3, 24936-24940.

18. Hanay, S. B., Brougham, D. F.,Dias, A. A., Heise, A., Polym. Chem., 2018, 8, 6594-6597

19. Hanay, S. B., O’Dwyer, J., Kimmins, S. D., de Oliveira, F. C. S., Haugh, M. G., O'Brien, F. J., Cryan, S. A., Heise, A., ACS Macro Lett., 2018, 7, 8, 944-949

20. Vonhören, B., Roling, O., De Bruycker, K., Calvo, R., Du Prez, F. E., Ravoo, B. J., ACS Macro Lett., 2015, 4 (3), 331-334

21. Billiet, S.; De Bruycker, K.; Driessen, F.; Goossens, H.; Van Speybroeck, V.; Winne, J. M.; Du Prez, F. E, Nat. Chem. 2014, 6, 815-821.

22. Kjell, D.P., Sheridan, R. S., J. Am. Chem. Soc. 1984, 106, 53685370

23. Houck, H. A., Blasco, E., Du Prez, F. E., Barner-Kowollik, C., J. Am. Chem. Soc. 2019, 141, 31, 12329-12337

24. De. Bruycker, K., Billiet, S., Houck, H. A., Chattopadhyay, S., Winne, J. M., Du Prez, F. E., Chem. Rev., 2016, 116, 3919-3974 